\title{
Short- and Long-Term Impact of a Multifaceted Approach Targeting Fluoroquinolone use in a Tertiary, Non- Teaching Hospital
}

Brianna Belsky ( $\sim$ brianna.belsky@hcahealthcare.com )

TriStar Skyline Medical Center https://orcid.org/0000-0001-6210-5770

Quentin Minson

TriStar Skyline Medical Center

\section{Research Article}

Keywords: Fluoroquinolone, Antibiotic, Antimicrobial stewardship, Interrupted time-series analysis, Enabling restriction, Community hospitals

Posted Date: January 10th, 2022

DOI: https://doi.org/10.21203/rs.3.rs-788992/v1

License: (c) (i) This work is licensed under a Creative Commons Attribution 4.0 International License. Read Full License 


\section{Abstract}

Background. While various strategies for antibiotic restrictions have been validated, their impacts are not well described in smaller, non-teaching facilities. Fluoroquinolones are an appropriate target for restriction based on their propensity for overuse and potential for causing "collateral damage."

Aim. Evaluate the impact of a multifaceted approach to decreasing fluoroquinolone use on fluoroquinolones and alternative antibiotics at a smaller, non-teaching facility.

Method. Retrospective, interrupted time series analysis conducted at a single 288-bed, tertiary, non-teaching hospital with 71 adult ICU beds comparing antibiotic consumption measured monthly by defined daily doses per 1000 adjusted patient days (DDD/1000 APD) prior to intervention (January 2011 to August 2014) to short-term (October 2014 to December 2015) and long-term (January 2018 to December 2019) periods following intervention.

Results. An increase in downward trends of fluoroquinolone use was observed from prior to intervention (-0.49 $\mathrm{DDD} / 1000 \mathrm{APD})$ to the short-term period (-1.13 DDD/1000 APD) and to a greater extent in the long-term period following the intervention (-1.32 DDD/1000 APD). Fluoroquinolone consumption decreased from 100.20 DDD/1000 APD in August 2014 to 73.96 DDD/1000 APD in the short-term and 14.89 DDD/1000 APD in the longterm intervention period. Levofloxacin susceptibility for Pseudomonas aeruginosa increased from $61 \%$ in 2014 to 83\% in 2018. No deleterious effects on Pseudomonas aeruginosa susceptibilities were observed for alternative antibiotics.

Conclusion. A multifaceted approach to decreasing fluoroquinolone use at a smaller, tertiary, non-teaching hospital led to a sustained decrease in consumption and a substantial increase in levofloxacin susceptibility to Pseudomonas aeruginosa.

\section{Impacts On Practice}

- In resource-limited settings such as smaller community hospitals, implementing pharmacist-driven multifaceted antimicrobial stewardship interventions can produce immediate and sustained impacts on antimicrobial consumption.

- Sustained antimicrobial stewardship initiatives targeting fluoroquinolones can lead to improvement in longterm exigencies such as Pseudomonas aeruginosa susceptibility rates without deleterious effects on alternative antibiotics.

\section{Introduction}

Antibiotic restriction, a strategy of antimicrobial stewardship (AMS), is a recognized as a primary intervention to decrease inappropriate antibiotic use [1]. Various strategies for antibiotic restriction exist, including prospective audit with intervention and feedback (PAF) and formulary restriction with preauthorization [2]. PAF is described as an enabling or persuasive strategy that provides more flexibility for the prescriber. It facilitates continuous engagement between the healthcare provider and the antibiotic stewardship program (ASP), which also makes it more labor intensive. Formulary restriction is described as a restrictive strategy that has been associated with a more immediate impact on antibiotic prescribing with less labor from the ASP but also a potential for redirecting antibiotic consumption to alternatives. A meta-analysis of 29 interrupted time series (ITS) analyses of restrictive interventions in a Cochrane review demonstrated that incorporating an enabling component, which was included in 
$13(45 \%)$ of the studies, consistently enhanced the effect of the interventions ( $+38.36 \%$; $95 \% \mathrm{Cl}, 18.94$ to $57.78 \%$ ) [3]. Current guidelines by the Infectious Diseases Society of America and Society for Healthcare Epidemiology of America recommend including either strategy or a combination of both strategies depending on the availability of facility-specific resources [1]. Determination of which strategy or combination of strategies will be most appropriate at a practice site is multifactorial including availability of formulary and non-formulary agents, infrastructure, and perceived urgency of the need for intervention [4-6]. For example, it might not be feasible for a facility without 24hour infectious disease (ID) coverage to be able to utilize a strict preauthorization strategy, as it could lead to delays in therapy.

Fluoroquinolone use has been demonstrated to be a risk factor for multidrug resistance (MDR) in bacteria, which can result in higher hospital costs [7, 8]. Fluoroquinolones can induce various antibiotic mechanisms of resistance (e.g., hyperexpression of MDR efflux systems in $P$. aeruginosa), thus potentially increasing resistance to other classes of antibiotics as well [7,9-10]. Previous studies at larger teaching hospitals have shown that restricting fluoroquinolones can lead to reversals in resistance of various bacteria and decrease superinfections [8, 11]. Boel et al showed that the implementation of an ASP at a 736-bed teaching hospital reduced the use of cephalosporins and fluoroquinolones, concomitantly decreasing levels of Escherichia coli at the intervention hospital compared to the control hospital. The combination of persuasive and restrictive strategies utilized during this study resulted in the intervention hospital experiencing a significant change in use of cephalosporins [2151 DDD/1000 bed-days (95\% Cl, 2177 to 2126$)$ ] and fluoroquinolones (244.5 DDD/1000 bed-days ( $95 \% \mathrm{Cl}, 258.9$ to 230.1)] [11]. The impact of persuasive and restrictive strategies on antibiotic consumption and susceptibility is not as well described in smaller, tertiary, non-teaching facilities.

Aim

The purpose of this study was to evaluate the immediate and lasting impacts of a multifaceted approach to decreasing fluoroquinolone use which was implemented in this type of setting in an effort to decrease hospitalonset Clostridioides difficile superinfections and improve $P$. aeruginosa susceptibility rates.

\section{Ethics approval}

The University of Tennessee Health Sciences Center (UTHSC) institutional review board approved this study as NHSR (Not Human Subjects Research) under identification number 20-07805-NHSR on January 27, 2021 prior to study initiation. All analyzed data pre-existed from routine antimicrobial stewardship protocols. No additional patient data was collected for the purpose of this study.

Setting

The study was conducted at a 288-bed, tertiary, nonteaching hospital with 71 adult intensive care unit (ICU) beds in Nashville, Tennessee. The ASP includes an ID physician and ID pharmacist during weekday daytime hours. On-site verifying pharmacist coverage extends to 24 hours every day.

\section{Method}

In September 2014, a multifaceted fluoroquinolone restriction was implemented. Measures included removing fluoroquinolones as antipseudomonal double coverage agents in healthcare-associated pneumonia (HCAP), hospital-acquired pneumonia (HAP), and ventilator-acquired pneumonia (VAP) order sets, relegating 
fluoroquinolones to "Alternative Therapy" status in community-acquired pneumonia (CAP) order sets, advising that fluoroquinolones be considered alternative agents in treatment of urinary tract infections (UTIs), and adding computerized physician order entry (CPOE) orders for shorter courses of UTI treatment. Education, updated fluoroquinolone toxicity data, institutional $C$. diff infection rates, and institutional fluoroquinolone susceptibility rates was provided to all relevant prescribers along with implementation of the restriction. In addition, restriction criteria were included in an ASP educational packet that is provided to all new prescribers and redistributed annually with updated (e.g., antibiogram) data. No restriction was placed on disciplines that could prescribe fluoroquinolones.

A retrospective, interrupted time series (ITS) analysis was conducted to review the impact of the fluoroquinolone restriction on usage trends over a 9-year span. Monthly antibiotic consumption was derived from hospital purchasing data and measured using defined daily doses per 1000 adjusted patient days (DDD/1000 APD) according to World Health Organization standards which did not change over the course of the study [16]. APD, number of patients times their lengths of stay plus estimated outpatient days of care, was utilized according to facility measurement protocols. Annual antibiogram susceptibility data was utilized to assess trends in antimicrobial susceptibility. In addition, medication usage evaluations (MUEs), which were performed periodically by the ASP throughout the studied period, were utilized to assess appropriateness of fluoroquinolone use and adherence of prescribers to the protocol. The pre-intervention period spanned from January 2011 through August 2014. The post-intervention period was divided into immediate and long-term periods to facilitate assessment of short-and long-term trends of fluoroquinolone consumption. The immediate post-intervention period spanned from October 2014 through December 2015, and the long-term post-intervention period spanned from January 2018 to December 2019. The primary endpoint was trend in fluoroquinolone consumption. Secondary endpoints included impact on consumption of alternative classes of antibiotics and susceptibility rates of $P$. aeruginosa.

\section{Statistical Analysis}

An interrupted time series model was utilized to evaluate the primary outcome. The pre-intervention period consisted of 44 data points, while the early post-intervention period and late post-intervention period had 15 and 25 data points, respectively. The model can be formulated as follows:

$Y_{t}=\beta_{0}+\beta_{1} *$ time $_{t}+\beta_{2} *$ intervention $_{t}+\beta_{3} *$ time $_{t} *$ intervention $_{t}+\varepsilon_{t}$

Where $Y_{t}$ is the fluoroquinolone use in DDD/1000 APD at time $t$, time is a continuous variable indicating time in months at time $t$ from the start of the study, intervention is an indicator for time $t$ occurring before or after the implementation of the restriction policy, and time * intervention is an interaction term. In this model, $\beta_{0}$ is the intercept estimating the baseline level for the beginning of the time series, $\beta_{1}$ estimates the slope before the intervention, $\beta_{2}$ estimates the intercept change in DDD/1000 APD after the intervention, $\beta_{3}$ estimates the change in slope for times after the intervention, and $\varepsilon_{t}$ is random error.

For the secondary outcome of alternative antibiotic consumption, DDD/1000 APD the three specified periods were compared in a pair-wise fashion using a Wilcoxon Rank Sum test. The other secondary outcome of changes in susceptibility of $P$. aeruginosa to fluoroquinolones was analyzed using Chi-square tests.

\section{Results}


The trend in fluoroquinolone consumption from the pre-intervention period to the immediate post-intervention period is depicted in Figure 1 [Insert Figure 1 here]. Fluoroquinolone consumption in August 2014, the month immediately preceding the intervention, was 100.20 DDD/1000 APD. In October 2014, the month immediately following the implementation, consumption decreased to 73.96 DDD/1000 APD. By the end of the immediate postintervention period in December 2015 (15 months after implementation), fluoroquinolone use had declined by more than half to 48.13 DDD/1000 APD. Our linear regression model comparing the pre-intervention period to the immediate post-intervention period is as follows:

$$
Y_{t}=165.37+-0.49 * \text { time }_{t}+--46.90 * \text { intervention }_{t}+-0.64 * \text { time }_{t} * \text { intervention }_{t}
$$

Of note, in the pre-intervention period, there was already a significant downward trend in usage $\left(\beta_{1}\right)$, such that each month usage dropped by approximately -0.49 DDD/1000 APD $(p=0.0255)$. In the immediate post-intervention period, there was an increase in the downward trend in usage $\left(\beta_{1}+\beta_{3}\right)$, with fluoroquinolone use reduced by approximately - 1.13 DDD/1000 APD per month $(p=0.0392)$.

The trend in fluoroquinolone consumption from the pre-intervention period to the long-term post-intervention period is depicted in Figure 2 [Insert Figure 2 here]. There was a sustained downward decline resulting in a dramatic sizeable decrease in fluoroquinolone consumption from 100.20 DDD/1000 APD in August 2014 to 14.89 DDD/1000 APD in December 2019 (64 months after implementation), as well as a significant change in usage seen during the long-term post-intervention period from 48.68 DDD/1000 APD in January 2018. This long-term post-intervention downward trend $\left(\beta_{1}+\beta_{3}\right)$ was more pronounced than the immediate post-intervention period, with fluoroquinolone use decreasing by approximately -1.32 DDD/1000 APD per month ( $<$ 0.0001). Our linear regression model comparing the pre-intervention period to the long-term post-intervention period is as follows:

$$
Y_{t}=165.37+-0.49 * \text { time }_{t}+-64.07 * \text { intervention }_{t}+-0.83 * \text { time }_{t} * \text { intervention }_{t}
$$

The impact of the fluoroquinolone restriction policy on the consumption of other antibiotics is shown in Table 1. It shows the median monthly usage of the antibiotics during the three time periods. Significant upward trends in usage were observed for antipseudomonal beta-lactams and specifically cefepime and group 2 carbapenems, as well as 3rd generation cephalosporins, macrolides, and tetracyclines from the pre-intervention period to the longterm post-intervention period. Significant downwards trends in usage when comparing these same periods were observed with piperacillin/tazobactam, aztreonam, aminoglycosides, nitrofurantoin, and sulfamethoxazole/trimethoprim. 
Table 1

Median monthly usage of antibiotics (DDD/1000 APD)

Median Monthly DDD/1000 APD

p-value

\begin{tabular}{|c|c|c|c|c|c|}
\hline Drug & $\begin{array}{l}\text { Pre- } \\
\text { Intervention }\end{array}$ & $\begin{array}{l}\text { Immediate } \\
\text { Post- } \\
\text { Intervention }\end{array}$ & $\begin{array}{l}\text { Long-term Post- } \\
\text { Intervention }\end{array}$ & $\begin{array}{l}\text { Pre vs. } \\
\text { Immediate } \\
\text { Post }\end{array}$ & $\begin{array}{l}\text { Pre vs. Long- } \\
\text { term Post }\end{array}$ \\
\hline Fluoroquinolones & 156.50 & 59.28 & 27.63 & $<0.0001$ & $<0.0001$ \\
\hline Piperacillin/Tazobactam & 52.06 & 38.54 & 31.16 & $<0.0001$ & $<0.0001$ \\
\hline Cefepime & 17.56 & 23.46 & 87.57 & 0.0079 & $<0.0001$ \\
\hline Carbapenems & 23.01 & 23.87 & 28.64 & 0.4123 & 0.0454 \\
\hline Aztreonam & 11.54 & 3.92 & 0.64 & $<0.0001$ & $<0.0001$ \\
\hline $\begin{array}{l}\text { Antipseudomonal } \\
\text { Beta-lactams }\end{array}$ & 102.45 & 86.77 & 145.20 & 0.0059 & $<0.0001$ \\
\hline $\begin{array}{l}\text { Cephalosporins } \\
\text { (2nd Generation) }\end{array}$ & 5.84 & 5.05 & 6.35 & 0.7806 & 0.4375 \\
\hline $\begin{array}{l}\text { Cephalosporins } \\
\text { (3rd Generation) }\end{array}$ & 45.67 & 53.74 & 66.27 & $<0.0001$ & $<0.0001$ \\
\hline Aminoglycosides & 13.19 & 10.70 & 8.74 & 0.0145 & $<0.0001$ \\
\hline $\begin{array}{l}\text { Macrolides } \\
\text { (Advanced Gen) }\end{array}$ & 26.57 & 42.61 & 52.48 & $<0.0001$ & $<0.0001$ \\
\hline Nitrofurantoin & 7.50 & 6.02 & 5.05 & 0.0033 & $<0.0001$ \\
\hline TMP/SMX & 9.54 & 9.71 & 6.50 & 0.9584 & 0.0003 \\
\hline Tetracyclines & 34.08 & 36.64 & 38.29 & 0.3700 & 0.0126 \\
\hline
\end{tabular}

Levofloxacin susceptibility rates to $P$. aeruginosa and other bacteria are shown in Table 2 [Insert Table 2 here]. There was a statistically significant increase in levofloxacin susceptibility for $P$. aeruginosa from the preintervention period (2011-2014) to the post-intervention period (2015-2018) (62\% vs. 76\%; $p<0.001)$. A statistically significant increase in levofloxacin susceptibility for Proteus mirabilis was observed (45\% vs. 55\%; P< 0.001$)$. The impact of the fluoroquinolone restriction policy on $P$. aeruginosa susceptibility rates to other antibiotics is depicted in Figure 4 [Insert Figure 4 here]. An increase in susceptibility to P. aeruginosa from 2014 to 2018 was seen in all antibiotics depicted. 
Table 2

Levofloxacin susceptibility to $P$. aeruginosa and other bacteria

\begin{tabular}{|llllllll|}
\hline \multicolumn{7}{|l|}{ Pre-Intervention (2010 - 2014) } & \multicolumn{3}{l|}{ Post-Intervention (2015 - 2018) } & \\
\hline Organism & $\begin{array}{l}\text { Total } \\
\text { Isolates }\end{array}$ & $\begin{array}{l}\text { Susceptible } \\
\text { Isolates }\end{array}$ & $\begin{array}{l}\text { Susceptible } \\
(\%)\end{array}$ & $\begin{array}{l}\text { Total } \\
\text { Isolates }\end{array}$ & $\begin{array}{l}\text { Susceptible } \\
\text { Isolates }\end{array}$ & $\begin{array}{l}\text { Susceptible } \\
\text { (\%) }\end{array}$ & $\begin{array}{l}\text { p-value } \\
\text { comparing } \\
\text { Pre and } \\
\text { Post }\end{array}$ \\
\hline $\begin{array}{l}\text { Pseudomonas } \\
\text { aeruginosa }\end{array}$ & 1,394 & 869 & $62 \%$ & 1,269 & 958 & $76 \%$ & $<0.001$ \\
\hline $\begin{array}{l}\text { Escherichia } \\
\text { coli }\end{array}$ & 8,517 & 5,605 & $66 \%$ & 9,180 & 6,136 & $67 \%$ & 0.147 \\
\hline $\begin{array}{l}\text { Enterobacter } \\
\text { clocae }\end{array}$ & 395 & 344 & $87 \%$ & 499 & 453 & $91 \%$ & 0.078 \\
\hline $\begin{array}{l}\text { Klebsiella } \\
\text { pneumonia }\end{array}$ & 1,728 & 1,681 & $97 \%$ & 2,259 & 2,178 & $96 \%$ & 0.124 \\
\hline $\begin{array}{l}\text { Proteus } \\
\text { mirabilis }\end{array}$ & 1,396 & 627 & $45 \%$ & 1,195 & 673 & $56 \%$ & $<0.001$ \\
\hline
\end{tabular}

Upon review of fluoroquinolone MUEs from 2014, 2015, and 2018-2020, all of which were retrospective reviews of fluoroquinolone use at our facility during the first week of March, there were significant decreases in the total number of fluoroquinolones prescribed across all indications since the implementation of the restriction as shown in Table 3 [Insert Table 3 here]. 
Table 3

Fluoroquinolone indication for the week of March 1-7 for 2014, 2015, 2018, 2019, 2020

\begin{tabular}{|c|c|c|c|c|c|}
\hline Indication & $\begin{array}{l}N(\%) \text { for } \\
2014\end{array}$ & $\begin{array}{l}N(\%) \text { for } \\
2015\end{array}$ & $\begin{array}{l}N(\%) \text { for } \\
2018\end{array}$ & $\begin{array}{l}N(\%) \text { for } \\
2019\end{array}$ & $\begin{array}{l}N(\%) \text { for } \\
2020\end{array}$ \\
\hline Total Courses & $62(100)$ & $53(100)$ & $32(100)$ & $20(100)$ & $9(100)$ \\
\hline Pneumonia & $34(55)$ & $17(32)$ & $9(28)$ & $8(40)$ & $3(33)$ \\
\hline CAP & $23(37)$ & $7(13)$ & $8(25)$ & $5(25)$ & $2(22)$ \\
\hline HAP/VAP & $11(17)$ & $8(15)$ & $1(3)$ & $3(15)$ & 0 \\
\hline COPD Exacerbation & $1(2)$ & $2(4)$ & $0(0)$ & $0(0)$ & $1(11)$ \\
\hline UTI & $21(34)$ & $21(40)$ & $5(16)$ & $2(10)$ & $2(22)$ \\
\hline Cholecystitis & $0(0)$ & $1(2)$ & $1(3)$ & $0(0)$ & $0(0)$ \\
\hline Surgical Prophylaxis & $2(3)$ & $5(9)$ & $1(3)$ & $4(20)$ & $0(0)$ \\
\hline $\begin{array}{l}\text { Cellulitis/Skin and Soft Tissue } \\
\text { Infection }\end{array}$ & $1(2)$ & $4(8)$ & $5(16)$ & $0(0)$ & $1(11)$ \\
\hline Osteomyelitis & $1(2)$ & $2(4)$ & $0(0)$ & $0(0)$ & $0(0)$ \\
\hline Intra-abdominal Infection & $2(3)$ & $4(8)$ & $4(13)$ & $4(20)$ & $2(22)$ \\
\hline Pyelonephritis/hydronephrosis & $0(0)$ & $2(4)$ & $6(19)$ & $1(5)$ & $1(11)$ \\
\hline Bronchitis & $0(0)$ & $0(0)$ & $0(0)$ & $1(5)$ & $0(0)$ \\
\hline Abscess & $0(0)$ & $1(2)$ & $0(0)$ & $0(0)$ & $0(0)$ \\
\hline Empiric (Evidence of Infection) & $2(3)$ & $0(0)$ & $1(3)$ & $0(0)$ & $0(0)$ \\
\hline
\end{tabular}

\section{Discussion}

The perceived urgency of the need to decrease $C$. difficile superinfections warranted a restrictive component to the fluoroquinolone intervention as determined by the ASP. Limited hospital resources including a lack of 24-hour ID coverage as well as the role of fluoroquinolones as an important non-beta-lactam antipseudomonal agent, however, precluded a more austere restriction. As a result, an immediate impact of the fluoroquinolone intervention indicative of a restrictive strategy was observed but not to the magnitude that might be expected from a strict preauthorization or formulary restriction. It is noteworthy that there was already a downward trend in fluoroquinolone usage in the 44 months immediately preceding the intervention. This likely reflects efforts by the ASP and prescribers in light of accumulating fluoroquinolone safety concerns including $C$. difficile. In contrast to more draconian restrictions, trends of decreasing fluoroquinolone prescribing increased in the short- and long-term periods, potentially owing to the continued reinforcement provided by the PAF component. A statistically significant decrease in fluoroquinolone consumption was observed during the long-term post-intervention period, indicating that the policy had a sustained impact. The ultimate result was a dramatic reduction of fluoroquinolone prescribing from 166.68 DDD/1000 APD in December 2011 to 14.89 DDD/1000 APD in December 2019.

The use of an ITS design is an increasingly popular method for evaluating the impact of AMS interventions allowing for statistical analysis of the magnitude of change resulting from an intervention both immediately and 
over time [12]. Our study had a sufficient number of data points to evaluate the effects over two separate time periods. Including a gap allowed for the evaluation of both short- and long-term trends of fluoroquinolone consumption.

The impact of the fluoroquinolone restriction policy on consumption of alternative classes of antibiotics was evaluated in order to elucidate the overall impact of the intervention on antibiotic usage (i.e., did we "squeeze the antibiotic balloon"). Shifts within the antipseudomonal beta-lactams were likely multifactorial including drug and IV fluid shortages and a similar aztreonam restriction implemented by the ASP during the study period. It is likely that the fluoroquinolone restriction contributed to the overall increase in antipseudomonal beta-lactam prescribing. It is likely that the significant upwards trend in usage of 3rd generation cephalosporins, macrolides, and tetracyclines is chiefly due to fluoroquinolones being relegated to "Alternative Therapy" status in CAP order sets as well as the impact of the sustained effort and educational campaign to reduce fluoroquinolone prescribing resulting in prescribers substituting these agents for chronic obstructive pulmonary disease (COPD) exacerbations.

$P$. aeruginosa susceptibilities were determined to be an appropriate microbiological marker given the clinical relevance and declining institutional susceptibility rates for levofloxacin. The increase in levofloxacin $P$. aeruginosa susceptibilities from $62 \%$ in 2014 to $76 \%$ in 2018 could have a significant clinical impact regarding its viability for empiric double-coverage as well as targeted therapy. Improvements in $P$. aeruginosa susceptibilities for antipseudomonal beta-lactams despite increased usage for most of these agents suggests that decreased levofloxacin usage may have been a contributing factor.

These results are consistent with that of Lafaurie et al, in which a sustained reduction of fluoroquinolone use (118.2 DDD/1000 PD to 77.5 DDD/1000 PD; P = 0.0002) following a primarily enabling intervention led to a significant decrease in fluoroquinolone-resistant $P$. aeruginosa $(42-26 \%$; $=0.001)$ in a 600 -bed acute care university hospital [8]. Sarma et al evaluated the effects of fluoroquinolone restriction on the resistance of Enterobacterales and observed a large decline in the percentage of ciprofloxacin-resistant ESBL-producing urinary E. coli isolates in both hospital (risk ratio, $0.473 ; 95 \% \mathrm{Cl}, 0.315$ to 0.712 ) and community settings (risk ratio, 0.098 ; $95 \% \mathrm{Cl}, 0.315$ to 0.712 ) and community settings (risk ratio, $0.098 ; 95 \% \mathrm{Cl}, 0.062$ to 0.157 ) [5]. Additionally, there were no observable increases in resistance of $E$. coli to other antibiotics as a result of their intervention.

Limitations of this study include its single-center setting and the retrospective nature of the analysis. Determination of whether the prescriber would have ordered a fluoroquinolone if not prohibited by the intervention can only be inferred. In 2016 and 2018, the Food and Drug Administration (FDA) issued Drug Safety Communication warnings associated with fluoroquinolones [13-14]. This is a potential confounder as it could have influenced fluoroquinolone prescribing practices. Antibiotic consumption was measured by DDD using purchasing data although current recommendations from the IDSA are to measure days of therapy (DOT) using antibiotic administrations as there can be dissimilarity between administered doses and the DDD recommended by the WHO as well as a lack of precision in utilizing purchasing data. We anticipate that this potential lack of precision should not have a profound impact on long-term effects as measured in our analysis [15].

In addition, other ASP interventions such as the aforementioned aztreonam restriction would be expected to have an indirect effect on fluoroquinolone prescribing. Despite these limitations, we feel that the results of this analysis can be impactful in demonstrating that ASP interventions combining restrictive and enabling components can be effective for the immediate determined need while also having sustained consequences, can be implemented in resource limited settings, and can lead to considerable improvements in susceptibilities. In conclusion, the 
multifaceted approach to targeting fluoroquinolone use at a tertiary, non-teaching hospital led to both an immediate and sustained impact on fluoroquinolone consumption, with only minor effects on consumption of alternative antimicrobials with improvements in $P$. aeruginosa susceptibility rates.

\section{Declarations}

Acknowledgments

The authors would like to acknowledge the support of Laurel Golding, MA for statistical analysis and Dr. Jayesh Patel, MD who is physician champion of the Antimicrobial Management Program.

Funding. The authors received no financial support for the research, authorship, and/or publication of this article.

Conflicts of interest. The authors declare no potential conflicts of interest with respect to the research, authorship, and/or publication of this article.

\section{References}

1. Barlam TF, Cosgrove SE, Abbo LM, et al. Implementing an antibiotic stewardship program: Guidelines by the Infectious Diseases Society of America and the Society for Healthcare Epidemiology of America. Clin Infect Dis 2016; e51-77. doi: 10.1093/cid/ciw118. Epub 2016 Apr 13. PMID: 27080992; PMCID: PMC5006285. Accessed June 29, 2021.

2. Chung GW, Wu JE, Yeo CL, et al. Antimicrobial stewardship: a review of prospective audit and feedback systems and an objective evaluation of outcomes. Virulence. 2013 Feb 15;4(2):151-7. doi: 10.4161/viru.21626. Epub 2013 Jan 9. PMID: 23302793; PMCID: PMC3654615.

3. Davey P, Marwick CA, Scott CL, et al. Interventions to improve antibiotic prescribing practices for hospital inpatients. Cochrane Database Sys Rev. 2017 Feb 9;2(2):CD003543. doi: 10.1002/14651858.CD003543.pub4. PMID: 28178770; PMCID: PMC6464541. Accessed June 29, 2021.

4. Claeys KC, Hopkins TL, Vega AD, et al. Fluoroquinolone Restriction as an Effective Antimicrobial Stewardship Intervention. Curr Infect Dis Rep. 2018 Mar 23;20(5):7. doi: 10.1007/s11908-018-0615-Z. PMID: 29572691.

5. Sarma JB, Marshall B, Cleeve V, et al. Effects of fluoroquinolone restriction (from 2007 to 2012) on resistance in Enterobacteriaceae: interrupted time-series analysis. J Hosp Infect. 2015 Sep;91(1):68-73. doi:10.1016/j.jhin.2015.05.006. Epub 2015 Jun 9. PMID: 26122624.

6. Shea KM, Hobbs ALV, Jaso TC, et al. Effect of a Health Care System Respiratory Fluoroquinolone Restriction Program To Alter Utilization and Impact Rates of Clostridium difficile Infection. Antimicrob Agents Chemother. 2017 May 24;61(6):e00125-17. doi: 10.1128/AAC.00125-17. PMID: 28348151; PMCID: PMC5444144.

7. Lewis GJ, Fang X, Gooch $M$, et al. Decreased resistance of Pseudomonas aeruginosa with restriction of ciprofloxacin in a large teaching hospital's intensive care and intermediate care units. Infect Control Hosp Epidemiol. 2012 Apr;33(4):368-73. doi: 10.1086/664763. PMID: 22418632.

8. Lafaurie M, Porcher R, Donay JL, et al. Reduction of fluoroquinolone use is associated with a decrease in methicillin-resistant Staphylococcus aureus and fluoroquinolone-resistant Pseudomonas aeruginosa isolation rates: a 10 year study. J Antimicrob Chemother. 2012 Apr;67(4):1010-5. doi: 10.1093/jac/dkr555. Epub 2012 Jan 11. PMID: 22240401. 
9. Werner NL, Hecker MT, Sethi AK, et al. Unnecessary use of fluoroquinolone antibiotics in hospitalized patients. BMC Infect Dis. 2011 Jul 5;11:187. doi: 10.1186/1471-2334-11-187. PMID: 21729289; PMCID: PMC3145580.

10. Poole K. Multidrug efflux pumps and antimicrobial resistance in Pseudomonas aeruginosa and related organisms. J Mol Microbiol Biotechnol. 2001 Apr;3(2):255-64. PMID: 11321581.

11. Boel J, Andreasen V, Jarløv JO, et al. Impact of antibiotic restriction on resistance levels of Escherichia coli: a controlled interrupted time series study of a hospital-wide antibiotic stewardship programme. J Antimicrob Chemother. 2016 Jul;71(7):2047-51. doi:10.1093/jac/dkw055. Epub 2016 Apr 7. PMID: 27055759.

12. Wagner AK, Soumerai SB, Zhang F, et al. Segmented regression analysis of interrupted time series studies in medication use research. J Clin Pharm Ther. 2002 Aug;27(4):299-309. doi: 10.1046/j.1365-2710.2002.00430.x. PMID: 12174032.

13. Hunt A. FDA in Brief: FDA warns that fluoroquinolone antibiotics can cause aortic aneurysm in certain patients. U.S. Food \& Drug Administration; 2018.

14. FDA Drug Safety Communication. FDA advises restricting fluoroquinolone antibiotic use for certain uncomplicated infections; warns about disabling side effects that can occur together. U.S. Food and Drug Administration; 2016.

15. Polk RE, Fox C, Mahoney A, et al. Measurement of adult antibacterial drug use in 130 US hospitals: comparison of defined daily dose and days of therapy. Clin Infect Dis. 2007 Mar 1;44(5):664-70. doi: 10.1086/511640. Epub 2007 Jan 22. PMID: 17278056.

16. WHO Collaborating Centre for Drug Statistics Methodology, Guidelines for ATC classification and DDD assignment. 2021. Oslo, 2020. ISBN 978-82-8406-165-8.

\section{Figures}

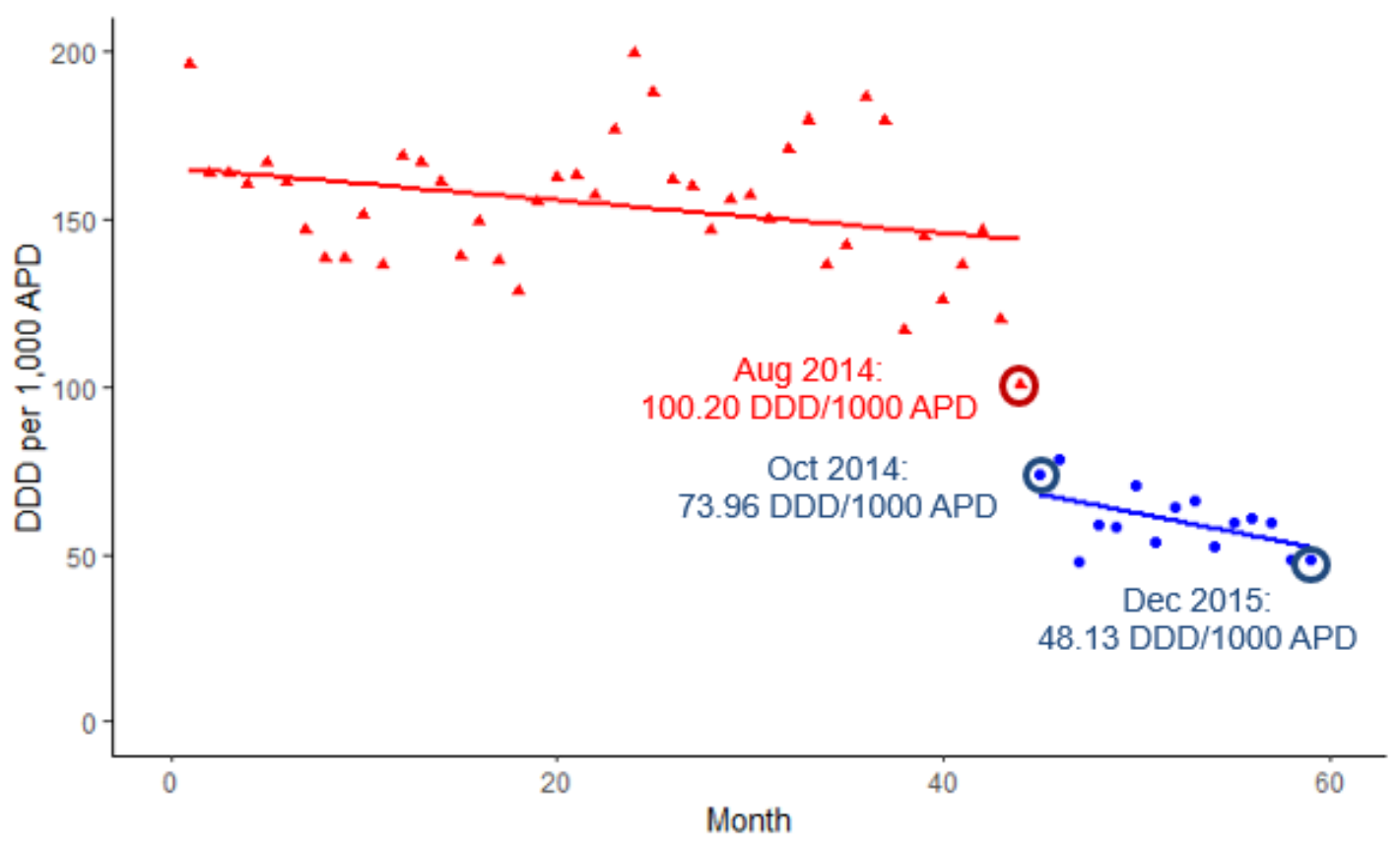

Time Period $\rightarrow$ Pre-Intervention $\rightarrow$ Post-Intervention Immediate

\section{Figure 1}


Comparison of fluoroquinolone usage pre-intervention (January 2011 to August 2014) and immediate postintervention (October 2014 to December 2015).

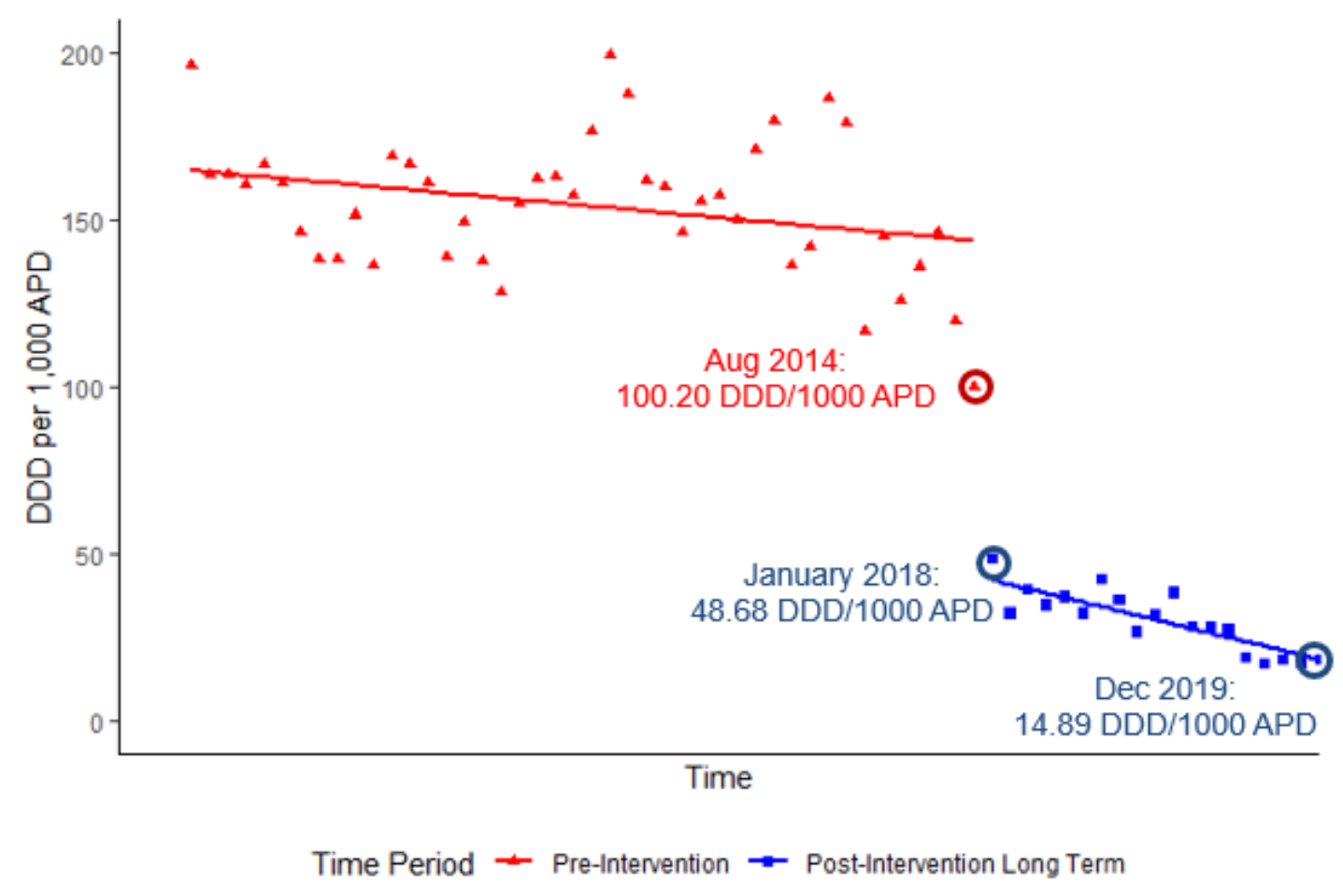

\section{Figure 2}

Comparison of fluoroquinolone usage in pre-intervention period (January 2011 to August 2014) and long-term post-intervention period (January 2018 to December 2019).

\section{Image not available with this version}

\section{Figure 3}

This image is not available with this version. 


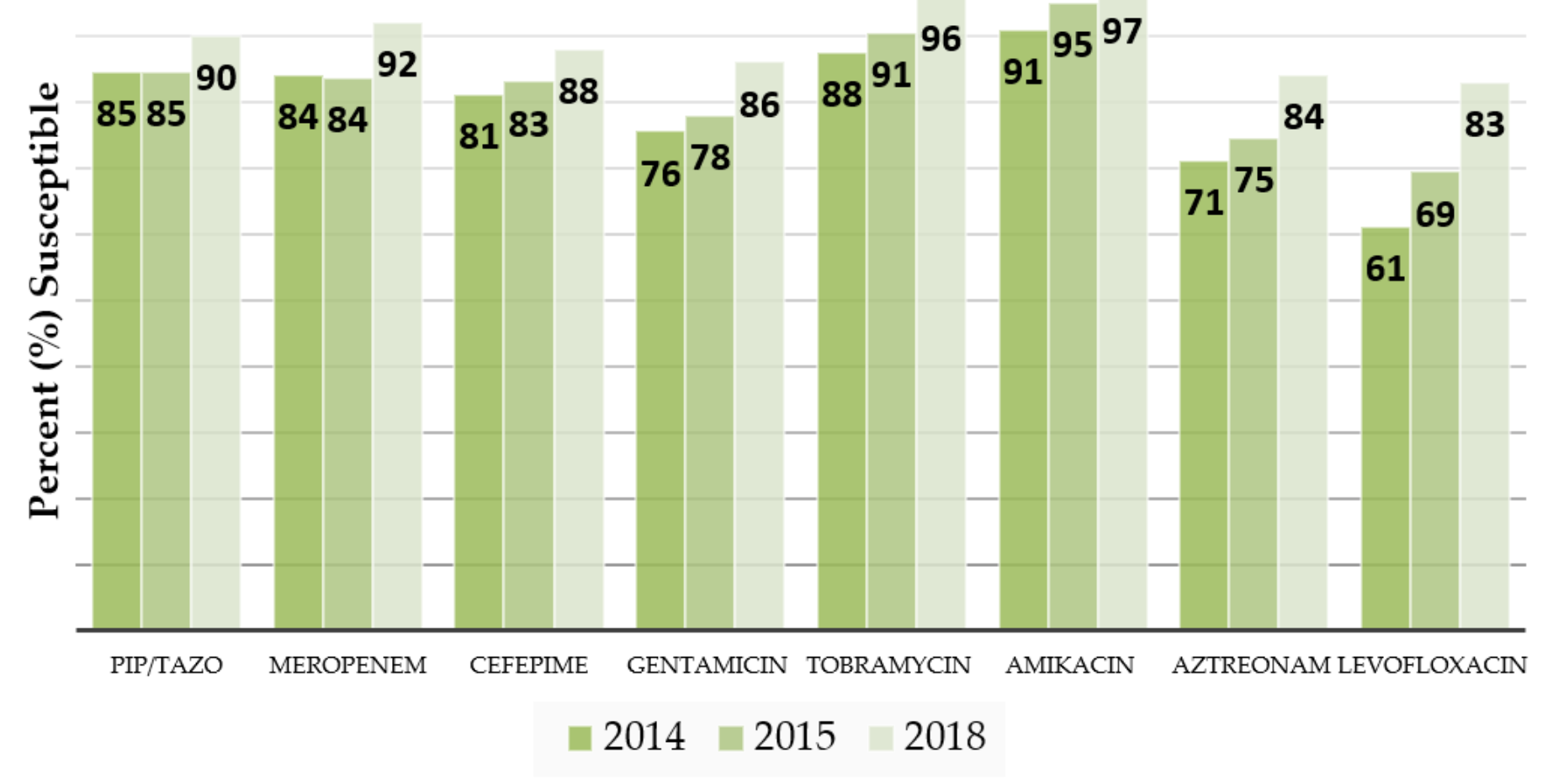

Figure 4

Pseudomonas aeruginosa susceptibility to levofloxacin and other antimicrobials

\section{Supplementary Files}

This is a list of supplementary files associated with this preprint. Click to download.

- STROBEStatement.pdf

- IJCPChecklist.pdf 